\title{
La autoría única en SciELO Chile: prácticas autoriales en las áreas de la ciencia
}

\author{
Paulina Meza \\ Omar Sabaj \\ Ken Matsuda \\ Universidad de La Serena - La Serena, Chile
}

\section{ARTICLE}

\begin{abstract}
Resumen
Dada la importancia del análisis de la cooperación, el estudio de la productividad científica se ha focalizado en las autorías múltiples y no ha abordado la autoría única. El propósito de este artículo es analizar las autorías únicas de la base SciELO Chile, entre los años 2010 y 2014. Los datos corresponden a 9.541 registros, con los cuales se estimó la proporción de la autoría única versus la multi-autoría, y el número de contribuciones por autor único en cada área de la ciencia. La autoría única representa el $29 \%$ del total publicado, y es una práctica frecuente en Humanidades y Ciencias Sociales. Por otra parte, los autores únicos, además, tienden a ser ocasionales, es decir, escriben solos y solo una vez. El estudio de la autoría única es fundamental para comprender las dinámicas particulares de la construcción de conocimiento científico y para generar e implementar políticas de investigación.
\end{abstract}

Palabras clave

Áreas de la ciencia; Autores únicos; Prácticas autoriales; Productividad científica; SciELO Chile.

\section{Single authorship in SciELO Chile: authorial practices in scientific areas}

\begin{abstract}
Given the importance of cooperation analysis, the scientific productivity study has been focused on multiple rather than single authorship. This article aims at analyzing single authorship in SciELO Chile between 2010 and 2014. The ratios of single to multiple authorship, and number of contributions to single authors for each scientific field were estimated using 9,541 entries. Single authorship, on the one hand, accounts for the $29 \%$ of total analyzed papers and is a usual practice in humanities and social sciences. On the other, single authors tend to be occasional, i.e., they write alone and only once. The study of single authorship is key to understanding the particular dynamics involved in the construction of scientific knowledge and to generating research policies.
\end{abstract}

Keywords

Authorial practices; SciELO Chile; Scientific areas; Scientific productivity; Single authors.

\section{Introducción}

La publicación de un artículo de investigación se ha transformado en una de las vías más importantes para validar y difundir el conocimiento. De allí que una de las formas de medir la productividad científica de un país radica en contabilizar la cantidad de artículos de investigación publicados por sus investigadores. En este contexto, la mayoría de los estudios cientométricos que abordan el tema de la productividad omiten un aspecto central, que representa un problema que afecta a los indicadores más populares que se utilizan para evaluar la productividad científica (e.g. número de artículos, número de citas, índice h, entre otros), y que tiene implicancias importantes en la asignación de fondos para la investigación (Taylor, 2015). 
Este aspecto ignorado, y tema de este artículo, es la autoría única, esto es, la práctica de escribir y publicar solo. A pesar de que se cuenta con algunas ideas preconcebidas respecto de qué disciplinas o áreas del conocimiento utilizan esta práctica más profusamente, no existen investigaciones que demuestren empíricamente estas ideas ni que describan cuán importante y representativa es esta práctica en las distintas áreas del conocimiento para explicar, así, su funcionamiento. Por otra parte, los pocos trabajos que sí toman en cuenta el tema de las autorías únicas solo consideran disciplinas o áreas de la ciencia particulares (generalmente las ciencias biológicas y de la salud), sin proporcionar datos de cómo varía esta práctica en todas las áreas de la ciencia (Molteni y Zulueta, 2002).

Históricamente, la autoría científica múltiple es una práctica muy reciente. Si se consensúa como fecha de aparición de las primeras revistas científicas el año 1665, las autorías múltiples no comienzan a aparecer sino hasta el final de la primera mitad del siglo XX (Bazerman, 1988). A pesar de ello, la autoría múltiple, como opuesta a la autoría única, ha sido objeto de mayor atención. Esto debido a dos razones principalmente. Primero, porque es un proxy para estudiar redes de cooperación, a partir de las redes de co-autorías; y segundo, porque ha surgido el tema ético de las autorías indebidas, esto es, preguntarse qué significa y quién merece ser autor o co-autor de una publicación científica (Salgado y Páez, 2007; Osborne y Holland, 2009; Valderrama, 2012).

El estudio de las autorías únicas (versus las autorías múltiples) es una dimensión muy importante para comprender las dinámicas particulares en las que cada disciplina construye el conocimiento científico. Así, el hecho de no considerar este indicador supone un problema metodológico operativo.

Diversos autores muestran sofisticados y exhaustivos estudios bibliométricos de la productividad de investigadores según las áreas de la ciencia, la afiliación y el país de afiliación (Molteni y Zulueta, 2002; Salgado y Páez, 2007; Buquet, 2013; Guerra-Pérez, 2013; Tarango y otros, 2015). Para ello, en estos trabajos, comúnmente se conforma una muestra de sujetos, se cuentan sus publicaciones y se agrupan de acuerdo a dichas variables. Para ilustrar el problema que se deriva de estos estudios, considérese un caso hipotético en el que, en la muestra analizada, se encuentra a un autor chileno titulado y ex académico de una universidad chilena que trabaja ahora en una universidad argentina y que ha publicado diez veces como autor único, una vez como co-autor de un artículo con un autor colombiano de otra disciplina y una vez como último autor de un trabajo con ocho autores de distintas partes del mundo y todos de disciplinas distintas. El sujeto, que posee un doctorado en economía, publica sus trabajos en revistas de derecho, economía e historia. Los problemas del análisis bibliométrico se reflejan en la dificultad de, ante estos datos, responder las siguientes preguntas: a) ¿Cuántas publicaciones tiene el sujeto?, b) ¿A qué disciplina pertenece?, c) ¿Cuál es su afiliación? y, d) ¿A qué país pertenece la publicación? Si bien el análisis de las autorías únicas no responde a todas estas preguntas, sí es una primera aproximación que permite dar cuenta de las prácticas disciplinares más específicas en las que se construye la ciencia. El dato final con el que comúnmente se cuenta a partir de este tipo de trabajos es que este sujeto tiene 12 publicaciones y que ellas pertenecen a tal o cual disciplina, afiliación o país, pero los criterios de asignación de cada categoría no son siempre explicitados. Este es un problema metodológico, pues existe una falta de definición de la productividad en términos operativos y porque no permite abordar los casos problemáticos.

Finalmente, cabe señalar que este trabajo es uno de los primeros intentos para dar cuenta de la productividad científica de SciELO Chile en todas las áreas de la ciencia, abarcando el período comprendido entre 2010 y 2014. Así, se puede dar cuenta de la diferencia entre autores únicos y múltiples, de las áreas de la ciencia más y menos productivas y de las instituciones que concentran en mayor y menor cantidad la productividad científica, asociada a la autoría única, de la base de datos mencionada.

\section{Métodos}

\subsection{Datos}

Con el objetivo de identificar, describir y analizar las autorías únicas en la librería electrónica SciELO Chile, como punto de partida, se utilizaron todos los registros aparecidos en esta base entre los años 2010 y 2014. Con la ayuda de un equipo de 3 asistentes entrenadas, se aplicaron dos criterios para delimitar los registros que se analizaron en este estudio: a) El género: Los registros a estudiar debían corresponder al género Artículo de Investigación (Sabaj y otros, 2010) y b) El idioma: como una forma de aislar esta variable, solo se consideraron 
trabajos publicados en español. Una vez aplicados estos criterios, se obtuvo un total de 9.541 artículos de investigación que constituyen los datos en estudio.

\subsection{Procedimientos de clasificación y análisis de los datos}

Sobre el total de los 9.541 registros se separaron los artículos escritos por un autor de aquellos escritos por más de uno. Con este dato, se obtuvo el porcentaje de autorías únicas en relación a las autorías múltiples en cada una de las áreas de la ciencia (ver Tabla 1). Una vez identificados los registros relevantes para la investigación, esto es, aquellos artículos escritos por un autor, se procedió a contabilizar el número de contribuciones, separando a los autores con una sola contribución de aquellos con más de una (ver Tabla 2).

Uno de los aspectos más problemáticos que se enfrenta a menudo en el estudio de la productividad científica es la adscripción de un trabajo a un área del conocimiento. Si bien en la plataforma SciELO cada revista aparece asociada a un área de la ciencia específica, su clasificación es muy débil, entre otras, por las siguientes razones: a) La clasificación se realiza a nivel de la revista y no del artículo, b) Una revista puede aparecer en más de un área de la ciencia, lo que, si bien es algo esperable debido al auge de la interdisciplinariedad científica actual, supone, para cualquier estudio que busque clasificar datos en áreas de la ciencia, tomar decisiones metodológicas que no siempre son explicitadas y, c) No todas las áreas de la ciencia propuestas por SciELO son áreas, sino que algunas son más bien conjuntos de disciplinas (Lingüística, letras y arte), por lo que, aquello general se traslapa con lo específico. Además, en este caso, se separa dicho conjunto de disciplinas de un área en la que podría tener cabida (Humanidades).

Ante esta problemática, se plantea un método propio de clasificación de publicaciones en distintas áreas de la ciencia. A diferencia de muchos estudios bibliométricos que centran su atención en el producto textual artículo de investigación (Hicks, 2009; Buquet, 2013; Mateo, 2015; Miguel y otros, 2015; Sivertsen, 2015), el interés de esta investigación se focalizó más bien en los sujetos que producen esos artículos y sus prácticas autoriales. Por ello, el tipo de clasificación propuesta se realizó sobre la base de la formación de los autores. Con este propósito, se efectuó una búsqueda de la información académica de todos los autores incluidos en la muestra, esto es, títulos, postítulos, grados académicos, ámbito de desarrollo de títulos y grados (Ingeniería, educación, filosofía, etc.), afiliación, proyectos de investigación, nacionalidad, cargos académicos, entre otros.

Luego del análisis de toda la información recopilada, se reclasificaron todos los autores de la muestra, según los criterios propuestos en esta investigación. Para ello, se utilizó la clasificación de áreas de la ciencia de la OCDE, tomando como criterio de ordenamiento el grado académico más alto obtenido por cada autor. En el caso de que algún sujeto tuviera dos grados equivalentes, pero de distintas áreas de la ciencia, se resolvió su asignación según el grado académico afín con su título o con otro grado académico. Por ejemplo, un autor con título de Abogado, Doctor en Historia y Doctor en Derecho, según sus grados académicos más altos, podría clasificarse en Ciencias sociales (Doctor en Derecho) o Humanidades (Doctor en Historia). Se optó por clasificarlo en Ciencias Sociales porque su pregrado también pertenece a esta área (Derecho).

Con estos procedimientos se pudo obtener las áreas del conocimiento a las que pertenecen los autores únicos. Además, el análisis de estos datos permitió diferenciar aquellos autores únicos que tienen solo una publicación en el período estudiado, de aquellos que tiene más de una. Por último, para hacer más específica la caracterización de las autorías únicas, los datos se agruparon también respecto de otras dos variables, a saber, la naturaleza de la institución a la que pertenecía el autor (Agencia nacional de investigación, centro de investigación, universidad, etc.) y al tipo de institución, en términos de instituciones estatales, privadas o mixtas (ver Tablas 3 y 4 ).

\section{Resultados y discusión}

En la Tabla 1, se muestran los resultados respecto de las prácticas autoriales en las distintas áreas del conocimiento: 
Tabla 1 - Autorías únicas versus autorías múltiples en las áreas de la ciencia

\begin{tabular}{|l|c|c|c|c|c|}
\hline \multicolumn{1}{|c|}{ Áreas de la ciencia } & Total & Autoría única & Autoría múltiple & $\%$ autoría única & \% autoría múltiple \\
\hline Ciencias Naturales & 1271 & 77 & 1194 & 6,06 & 93,94 \\
\hline Ingeniería y Tecnología & 971 & 28 & 943 & 2,88 & 97,12 \\
\hline Ciencias Médicas y de Salud & 3148 & 178 & 2970 & 5,65 & 94,35 \\
\hline Ciencias Agrícolas & 181 & 4 & 177 & 2,21 & 97,79 \\
\hline Ciencias Sociales & 2343 & 1303 & 1040 & 55,61 & 44,39 \\
\hline Humanidades & 1537 & 1151 & 386 & 74,89 & 25,11 \\
\hline \multicolumn{1}{|c|}{ Total } & 9451 & 2741 & 6710 & 29,00 & 71,00 \\
\hline
\end{tabular}

Fuente: Elaborada por los autores.

Tal como se presenta en la Tabla 1, en el panorama de la productividad científica de SciELO Chile lo más común son las autorías múltiples o coautorías (71\%). Ello muestra que existe una marcada preferencia por el trabajo colaborativo ante el trabajo individual. Ahora bien, si se pone el foco en el caso específico de cada área del conocimiento, el panorama cambia. Así, se advierte que en algunas áreas el trabajo individual es casi inexistente, mientras que en otras es lo más frecuente. En el primer caso se encuentran las áreas Ciencias Naturales, en la que los trabajos individuales alcanzan el 6,06\%; Ingeniería y Tecnología con un 2,88\% de autorías únicas; Ciencias Médicas y de Salud con un 5,65\% y Ciencias Agrícolas, que con un 2,21\% es la disciplina con menor porcentaje de trabajos individuales. Todas estas áreas, entonces, privilegian ampliamente el trabajo cooperativo por sobre el individual.

En otras áreas, en cambio, lo más frecuente es el trabajo individual por sobre el colaborativo, como ocurre en Ciencias Sociales y Humanidades, áreas en las que los trabajos de autorías únicas alcanzan el $55,61 \%$ y $74,89 \%$, respectivamente. Estos resultados son similares a los hallazgos de Molteni y Zulueta (2002), quienes, en el caso de Argentina, destacan la ausencia de colaboración en Humanidades, frente a una tasa de cooperación algo más elevada en Ciencias Sociales.

Estos resultados sugieren que en aquellas áreas en las que predominan las autorías múltiples, las competencias son más especializadas y están más distribuidas (Bazerman, 1988). Por tanto, no se concibe la investigación en solitario, pues, por ejemplo, un autor es el gestor de la idea, otro quien diseña el instrumento, otro el que realiza el experimento, otro el que escribe el artículo, etc. Esto confirma los resultados de Salgado y Páez (2001), quienes encontraron que en la revista Mapfre Medicina, los autores individuales solo representan un $10 \%$. Junto a lo anterior, en Ciencias Sociales y Humanidades, en cambio, donde predominan las autorías únicas, todo lo que se necesita para investigar y publicar, dada la naturaleza de la disciplina y de los objetos de estudio, pueden encontrarse en una sola persona. Estos resultados son coincidentes con los expuestos por Guerra-Pérez (2007) para el caso de dos disciplinas de las Ciencias Sociales (Bibliotecología y Ciencias de la información) en Cuba, donde más de un $60 \%$ de las autorías son individuales.

Por otro lado, también a partir de los resultados de la Tabla 1, se puede ver cuáles son las áreas más productivas en SciELO Chile. Así, el área que más aporta es Ciencias Médicas, que contribuye con 3148 de 9541 publicaciones, lo que equivale al $33,31 \%$ del total de la productividad científica de toda la base de datos analizada. Luego, el segundo y el tercer lugar son ocupados por aquellas áreas en las que las autorías únicas son una práctica muy frecuente, esto es, Ciencias Sociales, que contribuye con 2343 de 9541 publicaciones (24,79\%), mientras que Humanidades participa con 1537 del mismo total (16,26\%). Esto indica que más del 50\% de la productividad científica de SciELO Chile está concentrada en solo dos áreas, a saber, las Ciencias Médicas y las Ciencias Sociales.

Asimismo, resulta sorprendente que áreas como Humanidades y Ciencias Sociales estén entre los primeros lugares de la productividad científica, pues se ha señalado que son áreas en las que el conocimiento se difunde, principalmente, mediante otros tipos de documentos, distintos del artículo de investigación (monografías fundamentalmente) (Molteni y Zulueta, 2002). Quizás, es por esta misma razón que Ciencias Médicas presenta mayor cantidad de publicaciones, pues es una práctica común comunicar el conocimiento a través de revistas científicas y de artículos de investigación, específicamente. 
Ahora bien, a fin de realizar un análisis aún más detallado, en la Tabla 2, se presenta una caracterización de los autores únicos de cada área de la ciencia, señalando si son autores que, en el período estudiado, aportan con un solo artículo (autores con una contribución o autores ocasionales en palabras de Valera-Garrido y de-la-GalaSánchez, 2001) o con más de uno (autores con más de una contribución, i.e. productores medios y grandes productores). Estos resultados se presentan en términos de frecuencia absoluta (F.A.) y frecuencia relativa (\%).

Tabla 2 - Cantidad de contribuciones por autores únicos en cada área de la ciencia

\begin{tabular}{|c|c|c|c|c|}
\hline \multirow{2}{*}{ Áreas de la ciencia } & \multicolumn{2}{|c|}{ Autores con una contribución } & \multicolumn{2}{|c|}{ Autores con más de una contribución } \\
\hline & F.A. & $\%$ & F.A. & $\%$ \\
\hline Ciencias Naturales & 59 & 88,06 & 8 & 11,94 \\
\hline Ingeniería y Tecnología & 27 & 100 & 0 & 0 \\
\hline Ciencias Médicas y de Salud & 117 & 84,78 & 21 & 15,22 \\
\hline Ciencias Agrícolas & 4 & 100 & 0 & 0 \\
\hline Ciencias Sociales & 665 & 76,44 & 205 & 23,56 \\
\hline Humanidades & 646 & 77,46 & 188 & 22,54 \\
\hline Total & 1518 & 78,25 & 422 & 21,75 \\
\hline
\end{tabular}

Fuente: Elaborada por los autores.

Como se puede ver, en todas las áreas de la ciencia, en el período analizado, lo que predomina ampliamente en SciELO Chile son los autores que aportan con una contribución. De hecho, del total de esta base de datos, el $78,25 \%$ son autores que han realizado solo una contribución. Sin embargo, a través de las distintas áreas de la ciencia, existe una variación en razón de la proporción de autores con una o con más de una contribución. Así, se advierte que es en las disciplinas en las que lo más frecuente son las autorías únicas (Ciencias Sociales y Humanidades), donde existe mayor cantidad de autores que aportan con más de una contribución a su área. Más específicamente, en Ciencias Sociales los autores con más de una publicación alcanzan el 23,56\% y en Humanidades un $22,54 \%$.

En otras áreas, en cambio, no existen autores con más de una contribución, como ocurre con Ciencias Agrícolas e Ingeniería y Tecnología, donde el $100 \%$ de los autores únicos son ocasionales, pues aportan solo con una publicación a su área. Una posible explicación para ello radica en el hecho de que esta es un área en la que lo predominante son las autorías múltiples, por lo que escribir solo resulta extraño; en consecuencia, cuando un autor decide escribir solo, es un caso tan particular que, lo más probable, es que lo haga solamente una vez. Esta misma explicación es válida también para otras áreas en las que las autorías únicas son poco frecuentes, tales como Ciencias Naturales y Ciencias Médicas y de Salud. Por el contrario, en áreas en las que lo más frecuente son las autorías únicas, es probable que esos autores hagan más de una contribución a su área, como ocurre con Ciencias Sociales y Humanidades.

Tabla 3 - Autores únicos por tipo de institución en cada área de la ciencia

\begin{tabular}{|l|c|c|c|c|c|c|}
\hline \multicolumn{1}{|c|}{ Áreas de la ciencia } & ANI & CI & ES & U & NDA & Otros \\
\hline Ciencias agrícolas & 0 & 0 & 0 & 75 & 0 & 25 \\
\hline Ciencias médicas y de salud & 0,56 & 12,36 & 12,36 & 73,60 & 2,81 & 10,11 \\
\hline Ciencias naturales & 3,90 & 0,00 & 0,00 & 85,71 & 3,90 & 1,30 \\
\hline Ciencias sociales & 0,69 & 0,23 & 0,23 & 89,26 & 6,60 & 2,07 \\
\hline Humanidades & 1,30 & 0 & 0 & 93,31 & 3,30 & 1,04 \\
\hline Ingeniería y tecnología & 0 & 0 & 0 & 92,86 & 0,00 & 3,57 \\
\hline
\end{tabular}

Fuente: Elaborada por los autores.

Leyenda: $\mathrm{ANI}=$ Agencia Nacional de Investigación, $\mathrm{Cl}=$ Centro de Investigación, ES=Establecimientos de salud, U= Universidad, NDA= No declara afiliación, Otros= Academia, Asociación, corporación, empresas, fundaciones, institutos, investigadores independientes, organizaciones. 
Por otro lado, en la Tabla 3, se muestra el porcentaje de autores únicos por cada área de la ciencia según la naturaleza de la institución a la cual aparecen afiliados. Si bien para realizar el análisis se registró la afiliación específica de cada autor, para mostrar los resultados se agrupan dichas afiliaciones en cinco categorías: "Agencia Nacional de Investigación", "Centro de Investigación", "Establecimiento de salud", "Universidad", "no declara afiliación" y "otros" (donde se incluyen todas aquellas categorías que no tienen una presencia significativa).

Tal como se muestra en la Tabla 3, independientemente del área de la ciencia, los autores únicos de la base SciELO Chile se concentran en universidades. Esto es similar a lo descrito para España, donde la producción científica de las universidades comprende el 60,4\% de la producción científica total, utilizando sólo el 32\% del presupuesto de I+D (Salgado y Páez, 2007); lo mismo es reportado por Molteni y Zulueta (2002) para el caso de Argentina. En el caso de SciELO Chile, la producción científica de todas las áreas se concentra, con altísimos porcentajes (todos sobre el 70\%) en universidades. Solo en el área de las Ciencias Médicas y de salud, donde la autoría única es poco frecuente (menor a un 6\%, según los datos de la Tabla 1), las afiliaciones están más distribuidas, específicamente, en Establecimientos de Salud (12,36\%), Centros de Investigación (12,36\%) y Agencias Nacionales de Investigación (0,56\%).

En las Ciencias Sociales, donde prevalecen las autorías ocasionales, el panorama es similar, aunque con menos participación en otros tipos de instituciones diferentes a las universidades: Un 0,69\% de autores proviene de Agencias Nacionales de Investigación, un 0,23\% son autores afiliados a Centros de Investigación y un porcentaje igual está afiliado a Establecimientos de salud. Es en las Ciencias Sociales también donde se encuentra el mayor porcentaje de autores que no declaran afiliación. De la Tabla 3, es interesante observar, adempas, que las Ciencias Naturales y las Humanidades siguen un patrón similar donde priman las universidades y, en un bajo porcentaje, autores afiliados a agencias Nacionales de Investigación.

Por otro lado, se realiza una caracterización de la afiliación de los autores según su tipo, esto es, "institución estatal", "privada" y "estatal-privada"; se incluye también la categoría "otros" donde se agrupa a investigadores independientes y sin afiliación declarada. En la Tabla 4, se muestran, en términos porcentuales, los datos relativos a este aspecto:

Tabla 4 - Autores únicos por tipo de afiliación en cada área de la ciencia

\begin{tabular}{|l|c|c|c|c|}
\hline \multicolumn{1}{|c|}{ Áreas de la ciencia } & E & E/P & P tros \\
\hline Ciencias agrícolas & 75 & 0 & 25 & 0 \\
\hline Ciencias médicas y de salud & 62,36 & 0,56 & 34,27 & 2,81 \\
\hline Ciencias naturales & 72,73 & 0,00 & 23,38 & 3,90 \\
\hline Ciencias sociales & 39,75 & 0,15 & 53,49 & 6,60 \\
\hline Humanidades & 51,69 & 0,17 & 44,74 & 3,39 \\
\hline Ingeniería y tecnología & 60,71 & 0,00 & 39,29 & 0 \\
\hline
\end{tabular}

Fuente: Elaborada por los autores.

Leyenda: $E=$ Estatal, E/P= Estatal-privada, $\mathrm{P}=$ Privada, Otros: Investigadores independientes y sin afiliación declarada

Tal como se muestra en la Tabla 4, los tipos de afiliación de los autores únicos de la base SciELO Chile tienen un comportamiento disímil a través de las áreas de la ciencia. La afiliación estatal es la que prima en todas las áreas, a excepción de las Ciencias Sociales. Es interesante notar, además, que la afiliación a instituciones de origen estatal-privada son las menos frecuentes como tipos de afiliación de los autores únicos.

Las Ciencias Agrícolas y las Ciencias Naturales tienen una distribución similar de los datos, que comprende, de manera aproximada, unos tres cuartos de afiliaciones estatales versus un cuarto de afiliaciones privadas. El área de las Ciencias Médicas y de salud presenta un 62,36\% de autores pertenecientes a instituciones estatales y un $34,27 \%$ de autores asociados a instituciones privadas. El área de Ingeniería y Tecnología muestra una presencia muy similar a la de las Ciencias Médicas y de la salud con un $60,71 \%$ de autores afiliados a instituciones estatales y un $39,20 \%$ de autores de instituciones privadas. Por último, en las Humanidades, las 
afiliaciones estatales apenas pasan la mitad de la distribución (un 51,69\%) y existe una gran presencia de autores únicos afiliados a instituciones privadas (44,74\%).

El predominio de la responsabilidad investigativa de las instituciones estatales por sobre las privadas, sumado a los resultados de la Tabla 3, sugiere que, en general, la universidad estatal mantiene su función primera de ser la principal institución responsable de la generación de conocimiento. Ello podría justificarse en la necesidad de investigación existente en los países en desarrollo, por lo que el Estado debe financiar esta actividad como una forma de acortar la distancia que los separa de los países desarrollados. Otra explicación posible al predominio estatal en investigación se basa en el hecho de que, cada vez más, la productividad científica es una de las principales formas de medir la calidad de las instituciones financiadas por el estado (cantidad de publicaciones en revistas arbitradas, cantidad de citas de sus académicos, índice $\mathrm{H}$, entre otros).

A diferencia de todo el resto de las áreas de la ciencia, los autores únicos de las Ciencias Sociales se encuentran predominantemente afiliados a instituciones privadas, lo que podría explicarse por dos razones. Por una parte, las universidades privadas han desarrollado políticas de investigación que resultan atractivas para los investigadores de dicha área, cuya presencia ha aumentado de manera notable en esas instituciones. Por otro lado, ese aumento de investigadores de las Ciencias Sociales puede deberse a la necesidad de las instituciones privadas de posicionarse en esa área de la ciencia que tradicionalmente, en Chile, se ha desarrollado en el seno de universidades estatales.

\section{Conclusiones}

En este artículo se ha descrito la variación de las autorías únicas en la base SciELO Chile. Específicamente, se ha constatado que, si bien es una práctica común en Ciencias Sociales y en Humanidades, es muy poco frecuente en el resto de las áreas de la ciencia. Así también, se ha comprobado que los autores únicos en general son contribuidores ocasionales, esto es, solo aparecen con una publicación en el periodo estudiado. Esta tendencia cambia en el caso de las dos áreas de la ciencia donde la autoría única es más frecuente, a saber, Ciencias Sociales y Humanidades, donde los autores tienden a contribuir con más de una publicación en la base de datos estudiada. Asimismo, los datos mostraron una tendencia marcada de autores únicos afiliados a universidades estatales, a excepción de las Ciencias Sociales, para lo cual se han propuesto algunas interpretaciones.

Además de entregar un panorama general de la productividad científica en la base de datos SciELO Chile, el trabajo presenta un indicador, a saber, las autorías únicas, que no ha sido incorporado en estudios anteriores y que puede ser relevante para comprender las dinámicas particulares de la construcción de conocimiento científico.

Más específicamente, con este indicador se puede contribuir en la mejora de algunos indicadores bibliométricos que, en general, desconocen las características particulares de las prácticas autoriales en cada área de la ciencia, por lo que la comparación entre esas áreas, utilizando esos indicadores resulta inconducente (GómezCaridad y Bordons-Gangas, 1996).

Por último, dado que el comportamiento de las autorías únicas varía a través de las disciplinas, dicho indicador puede ser útil para que los encargados de las políticas públicas científicas generen estrategias diferenciadas para el financiamiento, según sean las prácticas autoriales propias de cada área.

\section{Agradecimientos}

Este artículo fue financiado por el proyecto FONDECYT de Postdoctorado N³150056 "El posicionamiento estratégico del autor en la comunicación del conocimiento científico: formas y funciones discursivas de la información propia y ajena en artículos de investigación”, en el cual se enmarca esta investigación. Agradecemos también a Verónica Aguilar, María Francisca Ríos y a Daniela Guerrero por su ayuda en la recolección de los datos. 


\section{Referencias}

Bazerman, C. (1988). Shaping Written Knowledge: The genre and activity of the experimental article in science. Madison: The University of Wisconsin Press.

Buquet, D. (2013). Producción e impacto de las ciencias sociales en América Latina. http://biblioteca.clacso.edu.ar/clacso/becas/20131016084109/Buquet Ciencias Sociales America Latina.pdf [Consultado en enero de 2016]

Gómez-Caridad, I. \& Bordons-Gangas, M. (1996). Limitaciones en el uso de los indicadores bibliométricos para la evaluación científica. Política Científica 46, 21-26.

Guerra-Pérez, M. (2007). Comportamiento de la productividad y la autoría en las revistas cubanas especializadas en Bibliotecología y Ciencia de la Información en el período 2000-2006. ACIMED 16 (6), 1.

Hicks, D. (1999). The difficulty of achieving full coverage of international social science literature and the bibliometric consequences. Scientometrics 44 (2), 193- 215.

Mateo, F. (2015). Producción científica en español en humanidades y ciencias sociales. Algunas propuestas desde Dialnet. El profesional de la información 24 (5), 509-515.

Miguel, S.; González, C. \& Chinchilla-Rodríguez, Z. (2015). Lo local y lo global en la producción científica argentina con visibilidad en Scopus, 2008-2012. Dimensiones nacionales e internacionales de la investigación. Información, cultura y sociedad 32, 59-78.

Molteni, V.; Zulueta, M. (2002). Análisis de la visibilidad internacional de la producción científica argentina en las bases de datos SSCl y A \& HCl en la década de 1990-2000: estudio bibliométrico. Revista Española de Documentación Científica 25 (4), 455-465.

Osborne, J.; Holland, A. (2009). What is authorship, and what should it be? A survey of prominent guidelines for determining authorship in scientific publications. Practical Assessment, Research \& Evaluation, 14 (15), 1-19.

Sabaj, O.; Matsuda, K.; Fuentes, M. (2010). Un modelo para la homogeneización de las clases textuales de la biblioteca electrónica SciELO-Chile: la variabilidad del artículo de investigación en diversas disciplinas. Información Tecnológica 21 (6), 133-148.

Salgado, J.; Páez, D. (2007). Scientific productivity and Hirsch's h index of Spanish social psychology: convergence between productivity indexes and comparison with other areas. Psicothema 19 (2), 179-189.

Sivertsen, G. (2015). Patterns of internationalization and criteria for research assessment in the social sciences and humanities. Scientometrics 107 (2), 357-368.

Tarango, J.; Hernández-Gutiérrez, P. \& Vázquez-Guzmán, D. (2015). Evaluation of scientific production in Mexican state public universities (2007-2011) using principal component analysis. El profesional de la información 24 (5), 567-576.

Taylor, M. (2015). Better ways to evaluate research and researchers. A SPARC Europe Briefing paper. http://sparceurope.org/wp-content/uploads/2015/12/Evaluate-SEBriefingPaper-1215.pdf [Consultado en enero de 2016]

Valera-Garrido, J. \& de-la-Gala-Sánchez, F. (2001). Análisis bibliométrico de la productividad científica en la revista MAPFRE MEDICINA". Mapfre Medicina, vol.12, pp.157-167.

Valderrama, J. (2012). Publicar en Revistas Científicas de Corriente Principal: Antecedentes, definiciones y recomendaciones. La Serena: Editorial de La Universidad de La Serena. 


\section{Datos de los autores}

Paulina Meza

Profesora e investigadora post-doctoral de la Universidad de La Serena. Departamento de Artes y Letras. pmeza@userena.cl

Omar Sabaj

Profesor e investigador de la Universidad de La Serena. Departamento de Artes y Letras.

omarsabaj@userena.cl

Ken Matsuda

Profesor e investigador de la Universidad de La Serena. Departamento de Matemáticas.

kmatsuda@userena.cl

Recibido - Received : 2016-07-04

Aceptado - Accepted: 2017-03-18

\section{$(\mathrm{cc}) \overline{\mathrm{EY}}$}

This work is licensed under a Creative Commons Attribution 4.0

United States License.

\section{ULLS D-Suret}

This journal is published by the University Library System of the University of Pittsburgh as part of its

D-Scribe Digital Publishing Program and is cosponsored by the University of Pittsburgh Press. 